\title{
A plane-wave pseudopotential description of charged clusters
}

\author{
F. Nogueira ${ }^{1}$, J.L. Martins ${ }^{2,3}$, and C. Fiolhais ${ }^{1}$ \\ ${ }^{1}$ Departamento de Física and Centro de Física Computacional, Universidade de Coimbra, 3000 Coimbra, Portugal \\ ${ }^{2}$ Departamento de Física, Instituto Superior Técnico, Avenida Rovisco Pais, 1, 1096 Lisboa, Portugal \\ ${ }^{3}$ INESC, Rua Alves Redol, 9, 1000 Lisboa Codex, Portugal
}

Received: 2 September 1998 / Received in final form: 5 November 1998

\begin{abstract}
One of the most efficient approaches in computational cluster physics uses a plane-wave basis set and pseudopotentials to describe electron-ion interactions. This method - where the clusters are placed inside supercells - is restricted in its usual form to neutral systems because of the long-range interaction between a charged cluster and its periodic images. To eliminate this restriction, we propose to shield each charged cluster with a spherical shell having a symmetric charge that neutralizes the supercell. Furthermore, the shell is placed in such a way that it cancels the electric dipole of the charged cluster. We present relaxed geometries and cohesive energies of $\mathrm{Na}_{N}^{+}, N=2-9$ and 21, obtained with Langevin quantum molecular dynamics. Our local density approximation structures are very similar to those found in other first principles calculations. Vertical and adiabatic ionization energies of $\mathrm{Na}_{N}, N=2,3,6$, and 8 are displayed. We also show results for $\mathrm{Na}_{8}^{2+}, \mathrm{Na}_{5}^{-}$and $\mathrm{Na}_{7}^{-}$.
\end{abstract}

PACS. 36.40.Wa Charged clusters - 31.15.Ar Ab initio calculations

\section{Introduction}

The calculation of the ground-state properties of complex systems is a challenging problem in material physics. In the Born-Oppenheimer (BO) approximation, the electrons feel an external potential due to the nuclei. The many-body problem is then reduced to a many-electron system under external forces. In density-functional theory, the total energy of the many-electron system is a functional of the single-particle electronic density, and its variational minimum with respect to the density would be the true groundstate energy if the exact functional were known. Minimizing the energy functional with respect to the density is equivalent to solving the Kohn-Sham equations. In this formulation, the unknown part of that functional is the exchange-correlation energy, for which the local density approximation (LDA) may be considered.

A practical approximation is to treat only the valence electrons, using pseudopotentials to represent their interaction with the ionic cores. This approach not only gives a more convenient energy scale but also removes the strong wiggles of the exact wave functions in the core region.

To solve the Kohn-Sham equations for infinite periodic systems, such as bulk solids, a plane-wave expansion of the wave functions is the natural choice [1]. This method has been widely used and optimized [2] and also allows for $a b$ initio calculations of nonperiodic systems such as atomic clusters. In fact, we may calculate physical properties of clusters as if they were a periodic system: We place them in a very large unit cell (supercell) in order to prevent the periodic images of the cluster from interacting with each other. The plane-wave basis set is orthonormal, and the convergence of the calculations increases systematically with the number of plane waves. Gaussian basis sets, on the other hand, do not provide a clear and systematic way to improve the convergence of the calculations and do not form an orthonormal set. As a result, the calculations often depend on the choice of basis set. Another advantage of plane waves is that the evaluation of forces for molecular dynamics is straightforward (the Pulay forces are identically zero). All these advantages have led to a considerable use of plane waves for ab initio calculations of finite systems [3].

However, this approach implies periodic boundary conditions and, therefore, spurious interactions between the periodic images of the system. The long-range interaction requires a large supercell to minimize this effect. In the case of a neutral system with a dipole moment, even bigger supercells are required, sometimes making the calculations impractical.

Charged clusters pose yet another problem, as they have a divergent Coulomb energy that must be removed. A common method is to neutralize the supercell through the introduction of a compensating jellium background [4]. This leads to spurious effects which are not always under control. A recent method [5] consists in using a cutoff in the Coulomb interaction. Here, we propose a new method to avoid the difficulties with charged systems: We place each image of the cluster within a spherical shell with total charge that neutralizes the supercell; if this shell is suitably positioned, the total electric dipole is zero and one is left with only quadrupolar interactions be- 
tween the periodic images of the charged cluster and its shell.

After a brief presentation of the method in Sect. 2, we present in Sect. 3 some of its results for charged sodium clusters and conclude in Sect. 4.

\section{Method}

The total energy in the pseudopotential Kohn-Sham formalism may be written as

$$
E[n]=T_{0}[n]+E_{\mathrm{xc}}[n]+E_{\mathrm{eI}}[n]+E_{\mathrm{Coul}}[n]+E_{\mathrm{II}},
$$

where $n(\mathbf{r})$ is the valence-electron density, $T_{0}$ is the kinetic energy of a noninteracting system with the same density as the real one, $E_{\mathrm{xc}}$ is the exchange-correlation energy, $E_{\mathrm{eI}}$ is the electron-ion interaction energy, $E_{\text {Coul }}$ is the Hartree energy of the valence electrons, and $E_{\mathrm{II}}$ is the ion-ion interaction energy. The electron-ion interaction energy can be written, for local pseudopotentials $w^{(i)}$, as

$$
E_{\mathrm{eI}}=\int \mathrm{d}^{3} \mathbf{r} n(\mathbf{r}) \sum_{m, i} w^{(i)}\left(\mathbf{r}-\mathbf{R}_{m}-\mathbf{t}_{i}\right),
$$

with $\mathbf{R}_{m}$ a Bravais lattice vector and $\mathbf{t}_{i}$ a vector locating ion $i$ within the unit cell. The Hartree energy is

$$
E_{\text {Coul }}=\frac{1}{2} \int \mathrm{d}^{3} \mathbf{r} \int \mathrm{d}^{3} \mathbf{r}^{\prime} \frac{n(\mathbf{r}) n\left(\mathbf{r}^{\prime}\right)}{\left|\mathbf{r}-\mathbf{r}^{\prime}\right|}
$$

and the ion-ion interaction energy is

$$
E_{\mathrm{II}}=\frac{1}{2} \sum_{(m, i) \neq\left(m^{\prime}, i^{\prime}\right)} \frac{Z_{i} Z_{i^{\prime}}}{\left|\mathbf{R}_{m}+\mathbf{t}_{i}-\mathbf{R}_{m^{\prime}}-\mathbf{t}_{i^{\prime}}\right|},
$$

with $Z_{i}$ the charge of the ion located at $\mathbf{t}_{i}$. Atomic units ( $\left.\hbar=1, m_{\mathrm{e}}=1, e^{2} / 4 \pi \epsilon_{0}=1\right)$ are used in this paper.

In a periodic system, the ion-ion energy is a divergent quantity. In reciprocal space, the Coulomb and pseudopotential contributions each diverge at the reciprocal lattice vector $\mathbf{G}=0$. If the system is neutral, the three divergent terms cancel each other, and their sum is simply the sum of two finite quantities $[1,2,6]: E_{\text {rep }}$, the repulsive part of the pseudopotential, given by

$$
E_{\mathrm{rep}}=\left(\sum_{i} \alpha_{i}\right)\left(\frac{1}{\Omega} \sum_{i} Z_{i}\right),
$$

with $\Omega$ the supercell volume, and

$$
\alpha_{i}=\frac{1}{\Omega} \int \mathrm{d}^{3} \mathbf{r}\left(w^{(i)}(\mathbf{r})+\frac{Z_{i}}{r}\right),
$$

and $E_{\text {Ewald }}$, the so-called Ewald energy [6]. The total energy in reciprocal space then becomes

$$
E=T_{0}[n]+E_{\mathrm{xc}}[n]+E_{\mathrm{eI}}^{\prime}[n]+E_{\text {Coul }}^{\prime}[n]+E_{\mathrm{rep}}+E_{\text {Ewald }},
$$
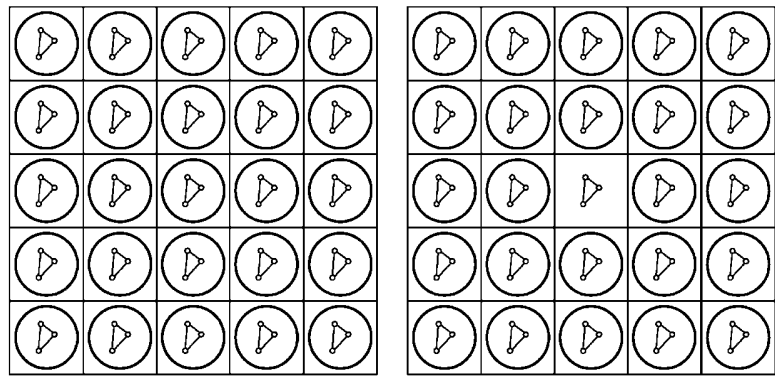

Fig. 1. Schematic illustration of the method used to calculate charged clusters: First, the periodic copies of the clusters are shielded from one another (left); then, the shielding is removed from one of the systems at the end of the self-consistent cycle (right).

where

$$
E_{\mathrm{eI}}^{\prime}=\Omega \sum_{i, \mathbf{G} \neq 0} n(\mathbf{G}) w^{(i)}(\mathbf{G}) e^{-i \mathbf{G} \cdot \mathbf{t}_{i}}
$$

and

$$
E_{\text {Coul }}^{\prime}=\frac{\Omega}{2} \sum_{\mathbf{G} \neq 0} \frac{4 \pi}{G^{2}}|n(\mathbf{G})|^{2} .
$$

$n(\mathbf{G})$ is the Fourier transform of the electronic density and $w^{(i)}(\mathbf{G})$ are the Fourier transforms of the pseudopotentials.

We may search for the equilibrium cluster geometry by molecular dynamics (MD) methods. These consist basically in solving classical equations of motion for the ions, with forces on them evaluated from the electronic structure (using the Hellman-Feynman theorem). This requires that the total energy gradient with respect to the ionic positions be calculated at each MD time step. Other local minimization procedures, like steepest descent methods, also require the computation of the gradient of (7).

To deal with charged clusters with a supercell method, we add a compensating charge with the form of a spherical shell. Since the system is neutral, all the above cancellations apply. If the shell is thin enough, the potential is constant in its interior, and the charged shell will not introduce spurious forces on the ions. Furthermore, if the shell is placed inside the supercell such as to cancel the dipole moment of the charged cluster, the cell-cell interactions will be at most quadrupolar, falling off as $r^{-4}$. At the end of the calculation, the spurious interaction energy between the shell and the charged cluster is subtracted (Fig. 1).

The charged shell can be simply inserted in a planewave code as a pseudopotential. A fake ionic species is introduced in the simulation, with a pseudopotential that emulates the potential created by the shell. The contribution of this ion to the total energy will amount to a constant, as long as the electronic density and the charged shell do not overlap. In this way, the introduction of the fake ion will simply shift the zero of the Kohn-Sham potential. To maintain the cancellation of the divergent terms, the repulsive part of this fake pseudopotential must be considered in (5). At the end of each self-consistency cycle, the 
interaction energy between the fake pseudopotential and the valence electrons has to be removed (if the shell is thin enough, this is the product of the shell potential at its center and the number of valence electrons). The fake ion will introduce spurious interactions in the Ewald energy and forces. We remove all those terms from the total energy and its gradient. For simplicity, only local potentials were considered in (2)-(9). Besides, as nonlocal terms arising from the use of nonlocal pseudopotentials are short-range, they do not affect the charge correction procedure.

\section{Results}

Sodium is the most studied of the simple metals, and this makes it the ideal test system. Its average valence-electron density is close to the equilibrium density of the jellium model, and the LDA is a very good approximation for the jellium surface problem [7].

In this section we present some results for Na clusters obtained with our method. Exchange and correlation effects are included with the Perdew and Zunger [8] parametrization of the Ceperley-Alder results [9]. For the shape of the charged shell we simply chose a Gaussian

$$
\varrho(r)=A e^{-(r-R)^{2} / d^{2}},
$$

with $A$ a constant chosen to give the desired total shell charge, $R$ the middle radius of the shell, and $d \ll R$ a convenient width. The radius of the shell of charge was chosen to be $R=12.7 \mathrm{bohr}$ and its width $d=0.21 \mathrm{bohr}$ (for $\mathrm{Na}_{21}^{+}$, we used $R=15.9 \mathrm{bohr}$ and $d=0.27 \mathrm{bohr}$ ). All the calculations were performed in an fcc supercell with lattice constant $a=40 \mathrm{bohr}$ (except $\mathrm{Na}_{21}^{+}$, for which we took $a=50 \mathrm{bohr}$ ). The plane-wave energy cutoff was $9 \mathrm{Ry}$. Convergence of the calculated cluster energies with both lattice constant and energy cutoff was better than $1 \mathrm{mRy}$. Only the $\Gamma$ point was considered when the reciprocal space was sampled, as is appropriate in a supercell calculation, and we used a Gaussian level broadening of 0.01 Ry [10]. We used the Troullier-Martins soft pseudopotential[11] with the cutoff radius $r_{\mathrm{c}}=2.6 \mathrm{bohr}$, and $s$ and $p$ nonlocality. The pseudopotential was cast into the Kleinman and Bylander separable form [12], with $l=1$ as the local potential.

In order to find the equilibrium positions $\left\{\mathbf{t}_{i}\right\}$ we used the Langevin quantum molecular dynamics (LQMD) method developed in [4]. This method is adequate for dealing with metallic clusters, since the dynamics is performed on the BO surface. The LQMD simulations started at $300 \mathrm{~K}$, the cluster being cooled down to $10 \mathrm{~K}$ in an exponentially decaying annealing schedule. The Langevin time step was 410 a.u. and the friction parameter 10 a.u. At the end of the LQMD run, a steepest descent optimization was performed.

As a first test, we calculated the equilibrium bond length of $\mathrm{Na}_{2}^{+}$, and found $d_{\mathrm{e}}=6.89 \mathrm{bohr}, 2.5 \%$ higher than the experimental value of $6.72 \mathrm{bohr}$ [13]. Part of this discrepancy is due to the use of LDA instead of the local spindensity approximation for the single electron. For the neu-
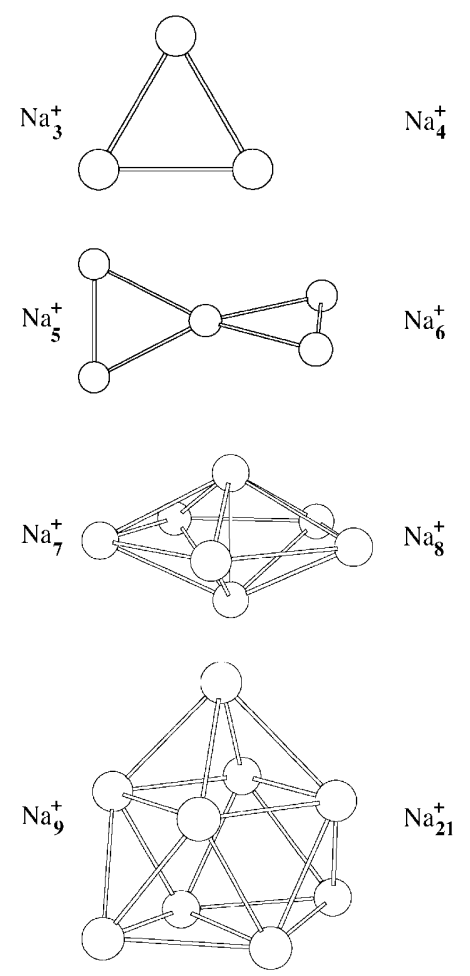

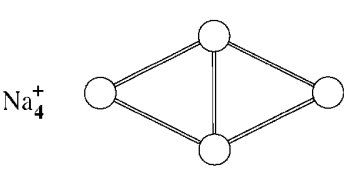

$\mathrm{Na}_{6}^{+}$

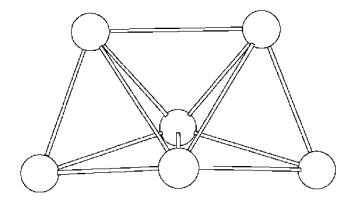

$\mathrm{Na}_{8}^{+}$
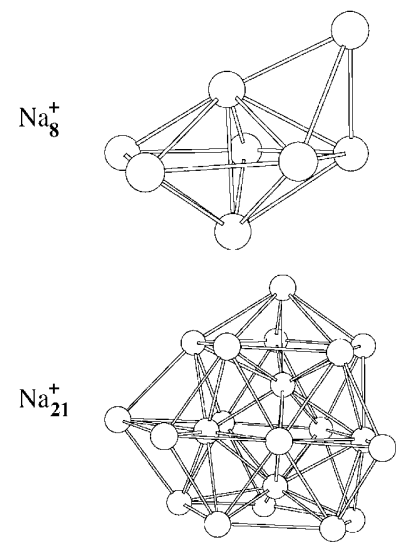

Fig. 2. Ground-state structures for $\mathrm{Na}_{N}^{+}$clusters with $N=$ $3-9$, and $21 . \mathrm{Na}_{3}^{+}, \mathrm{Na}_{9}^{+}$, and $\mathrm{Na}_{21}^{+}$are magic clusters.

tral dimer, we obtained $d_{\mathrm{e}}=5.68 \mathrm{bohr}$, in very good agreement $(-2.4 \%)$ with the experimental value 5.82 bohr [14].

We performed an unrestricted LQMD search for the minimum energy structures of $\mathrm{Na}_{N}^{+}$, with $N=3-9$ and 21 . The lowest-energy isomers are shown in Fig. 2. These structures are very similar to those obtained by quantum chemistry methods [15], which generally lead to an optical response that compares well with experiment.

Figure 3 shows the cohesive energies

$$
E_{\mathrm{c}}=\frac{(N-1) E(\mathrm{Na})+E\left(\mathrm{Na}^{+}\right)-E\left(\mathrm{Na}_{N}^{+}\right)}{N}
$$

for these structures. The values obtained are systematically higher than those obtained in the configuration interaction (CI) calculation shown for comparison. This is a manifestation of the over-binding characteristic of the LDA. However, the expected odd-even alternation in the cohesive energies is clearly seen in Fig. 3 . The cohesive energies increase more with cluster size than in the CI calculation.

Figure 4 shows the adiabatic and vertical ionization energies of the $\mathrm{Na}_{2}, \mathrm{Na}_{3}, \mathrm{Na}_{6}$, and $\mathrm{Na}_{8}$ clusters. The agreement with CI is fairly good, given that spin-polarization effects play a significant role in the ionization energies.

As an example of the applicability of the method to double-positively and negatively charged clusters, we show in Figs. 5 and 6 the ground-state structures of $\mathrm{Na}_{8}^{2+}, \mathrm{Na}_{5}^{-}$, and $\mathrm{Na}_{7}^{-} . \mathrm{Na}_{8}$ is the smallest cluster which can be doublepositively ionized in the present approach. In a jellium 


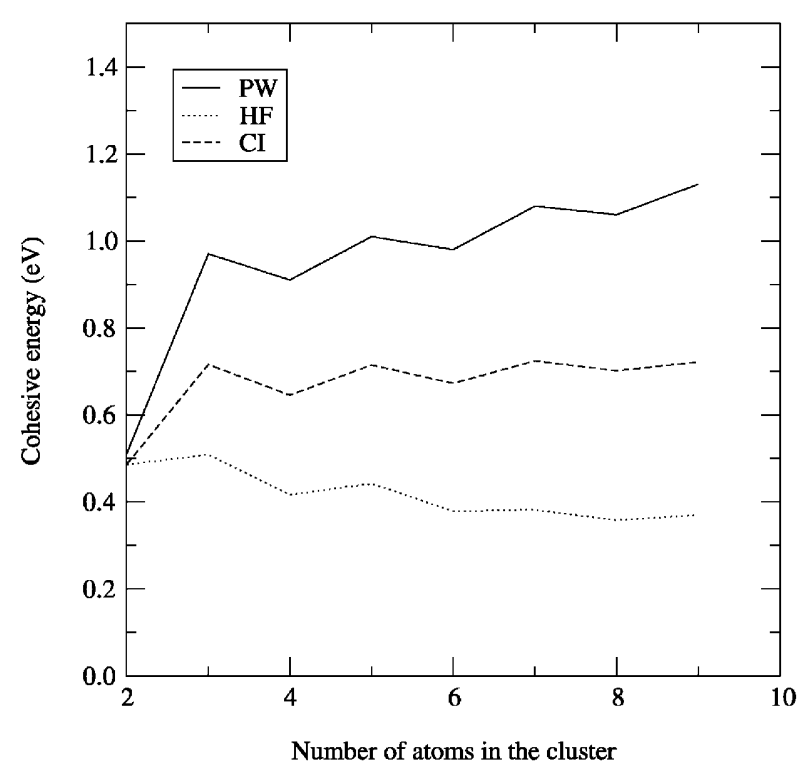

Fig. 3. Cohesive energies, in eV, for small cationic sodium clusters. Results are from the plane-wave method presented here (PW), and the configuration interaction (CI) [15] and HartreeFock (HF) [15] calculations.

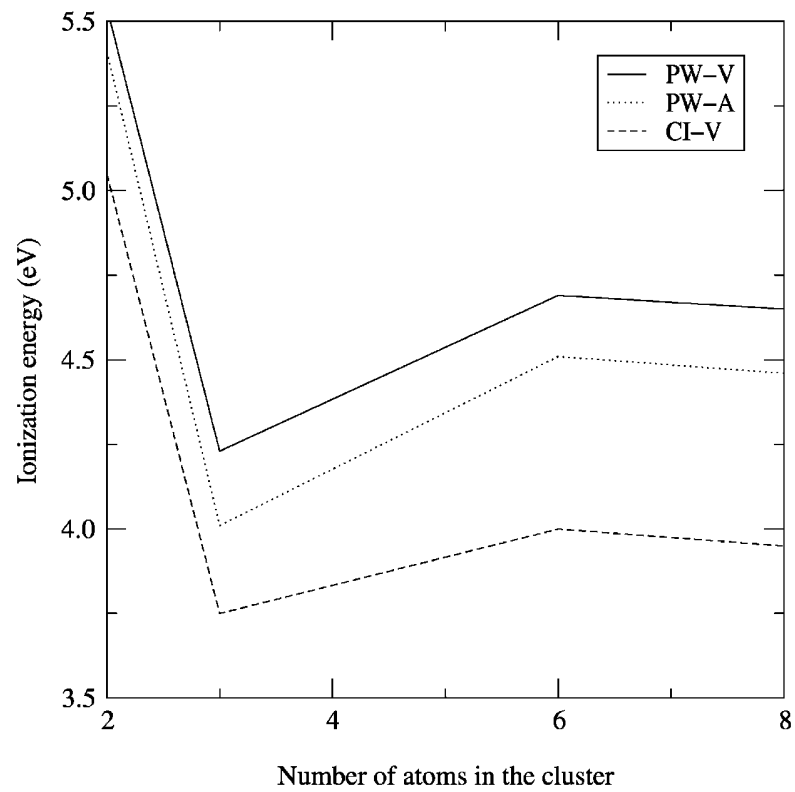

Fig. 4. Vertical (PW-V) and adiabatic (PW-A) ionization energies, in $\mathrm{eV}$, of the $\mathrm{Na}_{N}$ clusters with $N=2,3,6$, and 8 . The configuration interaction (CI-V) vertical ionization energies are from [17].

model, $\mathrm{Na}_{4}^{2+}$ is the smallest stable doubly ionized cluster [16]. The structure of $\mathrm{Na}_{8}^{2+}$ is much different from that of $\mathrm{Na}_{8}^{+}$. Its average bond length $(6.97 \mathrm{bohr})$ is larger than that of the singly ionized cluster ( $6.65 \mathrm{bohr})$ and that of the neutral cluster $(6.50 \mathrm{bohr})$. This charge-induced expansion was also found in a jellium model [16]. The equilibrium shape of $\mathrm{Na}_{5}^{-}$is planar, as was also found from CI calculations [17].

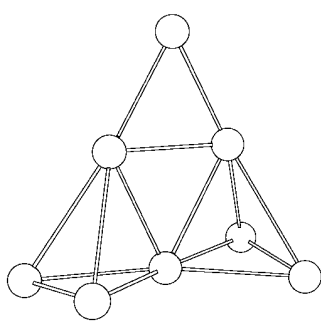

Fig. 5. Ground-state structure for $\mathrm{Na}_{8}^{2+}$.
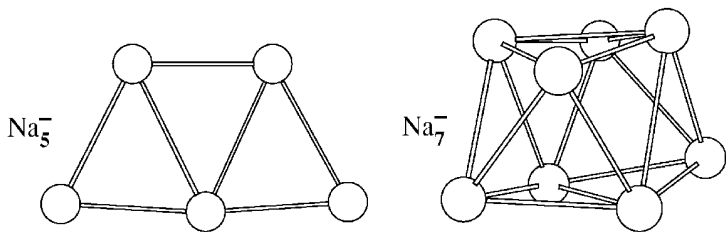

Fig. 6. Ground-state structures for $\mathrm{Na}_{5}^{-}$and $\mathrm{Na}_{7}^{-}$.

\section{Conclusions}

We introduced a novel method for ab initio simulations of finite charged systems in a plane-wave basis. This method allows one to deal accurately with positively or negatively charged clusters without artificial cutoffs or compensating jellium backgrounds that may affect the dynamics. Our LDA results are similar to those obtained in previous first principles calculations. With our method, we may study the stability of clusters with higher numbers of atoms and/or higher charges [18].

The LQMD minimization procedure employed efficiently samples the configuration space and can be used to perform finite-temperature simulations and evaluate thermodynamic and transport properties. In fact, cluster experiments are performed at some finite temperature. Temperature effects require further study.

We thank Dr. Jorge M. Pacheco for very fruitful discussions. This work has been partially supported by the PRAXIS XXI program (PRAXIS/2/2.1/FIS/26/94).

\section{References}

1. J. Ihm, A. Zunger, M.L. Cohen: J. Phys. C: Solid State Phys. 12, 4409 (1979)

2. W.E. Pickett: Comp. Phys. Rep. 9, 115 (1989)

3. U. Röthlisberger, W. Andreoni: J. Chem. Phys. 94, 8129 (1991)

4. N. Bingelli, J.L. Martins, J.R. Chelikowsky: Phys. Rev. Lett. 68, 2956 (1992)

5. M.R. Jarvis, I.D. White, R.W. Godby, M.C. Payne: Phys. Rev. B 56, 14972 (1997)

6. M.T. Yin, M.L. Cohen: Phys. Rev. B 26, 3259 (1982)

7. J.M. Pitarke, A.G. Eguiluz: Phys. Rev. B 57, 6329 (1998)

8. J.P. Perdew, A. Zunger: Phys. Rev. B 23, 5048 (1981)

9. D.M. Ceperley, B.J. Alder: Phys. Rev. Lett. 45, 566 (1980)

10. C. Elsässer, M. Fähnle, C.T. Chan, K.M. Ho: Phys. Rev. B 49, 13975 (1994) 
11. N. Troullier, J.L. Martins: Solid State Commun. 74, 613 (1990); N. Troullier, J.L. Martins: Phys. Rev. B 43, 1993 (1991)

12. L. Kleinman, D.M. Bylander: Phys. Rev. Lett. 48, 1425 (1982)

13. S. Martin, J. Chevaleyre, S. Valignat, J.P. Perrot, M. Broyer, B. Cabaud, A. Hoareau: Chem. Phys. Lett. 87, 235 (1982)

14. P. Kusch, M.M. Hessel: J. Chem. Phys. 68, 2591 (1978)
15. V. Bonačić-Koutecký, J. Pittner, C. Fuchs, P. Fantucci, M.F. Guest, J. Koutecký: J. Chem. Phys. 104, 1427 (1996)

16. F. Nogueira, A. Vieira, M. Brajczewska, C. Fiolhais: in Condensed Matter Theories, ed. by J. da Providência et al. (Nova Science, New York 1998)

17. V. Bonačić-Koutecký, P. Fantucci, J. Koutecký: Chem. Rev. 91, 1035 (1991)

18. R.N. Barnett, U. Landman, G. Rajagopal: Phys. Rev. Lett. 67, 3058 (1991) 